\title{
Percepção da População de Anápolis, Goiás sobre Dengue, Zika e Chikungunya
}

\author{
Perception of the population of Anápolis, Goiás about Dengue, Zika and Chikungunya
}

Percepción de la población de Anápolis, Goiás sobre Dengue, Zika y Chikungunya

Cláudio Henrique Martins de Souza ${ }^{1}$, Gabriela Lima Pereira', Geovanna Lemos Batista de Oliveira ${ }^{1 *}$, Letícia Maiara Nunes Araújoํㅜ, Mariana Sousa Lopes¹, Denis Masashi Sugita², Léa Resende Moura².

\section{RESUMO}

Objetivo: Verificar o nível de conhecimento da população de Anápolis-GO a respeito do vetor, aspectos clínicos e medidas preventivas de Zika, Dengue e Chikungunya, além de apontar quais meios de comunicação os entrevistados mais utilizam para encontrar tais conhecimentos. Método: Trata-se de um estudo transversal e descritivo realizado através de um questionário quali-quantitativo a partir de uma amostragem representativa. Foram entrevistados 432 moradores, de dezesseis bairros diferentes de Anápolis. Resultados: Foi encontrado uma população em sua maioria jovem e do sexo feminino, que soube apresentar a forma de transmissão comum às três doenças, porém o conhecimento do agente etiológico não foi satisfatório, havendo uma confusão entre o mesmo e o vetor. Quanto aos sinais e sintomas, observou-se maior conhecimento dos participantes sobre a Dengue e ainda um padrão de respostas similar para as três doenças o que denota a falta de distinção entre elas. Notou-se que a população de Anápolis conhece e tem aderido às formas de controle do mosquito Ae. Aegypti. Conclusão: Dessa forma, conclui-se que a população pesquisada possui conhecimento satisfatório sobre os locais de reprodução e as formas de prevenção do mosquito, mas esse conhecimento parece não refletir em suas atividades de prevenção em domicílio, o que contribui para o crescente número de casos dessas doenças. Logo, torna-se clara a necessidade de campanhas educativas e maior divulgação de informação sobre Dengue, Zika e Chikungunya que abordem não só as medidas preventivas como os agentes etiológicos, o ciclo da doença, seus sinais e sintomas e complicações.

Palavras-chave: Aedes, Saúde Pública, Comunicação em Saúde.

\begin{abstract}
Objective: Verify the level of knowledge of the population of Anápolis-GO regarding the vector, clinical aspects and preventive measures of Dengue, Zika and Chikungunya, besides pointing out which media the interviewees use most to find such knowledge. Methods: It is a cross-sectional and descriptive study conducted through a qualitative-quantitative questionnaire based on a representative sample of 432 residents of sixteen different neighborhoods of Anápolis. Results: It was found a population that knew how to present the common transmission form to the three diseases, but the knowledge of the etiological agent was not satisfactory, there being a confusion between the same and the vector. As for the signs and symptoms, there was a greater knowledge of the participants about Dengue and a similar pattern of responses for the three diseases, which indicates the lack of distinction between them. It was noticed that the population of Anápolis
\end{abstract}

\footnotetext{
${ }^{1}$ Acadêmico do curso de Medicina da UNIEVANGELICA, * E-mail: geovannabatista@yahoo.com.br

2 Professor do curso de Medicina da UNIEVANGÉLICA
} 
knows the forms of control of the mosquito Ae. Aegypti. Conclusion: Thus, it is concluded that the researched population has satisfactory knowledge about breeding sites and forms of mosquito prevention, but this knowledge does not seem to reflect in their prevention activities at home, which contributes to the increasing number of cases of these diseases. Therefore, it is clear the need for educational campaigns and greater dissemination of information on Dengue, Zika and Chikungunya that address not only the preventive measures as the etiological agents, the cycle of the disease, its signs and symptoms and complications.

Key Words: Aedes, Public Health, Health Communication.

\section{RESUMEN}

Objetivo: Verificar el nivel de conocimiento de la población de Anápolis-GO respecto al vector, aspectos clínicos y medidas preventivas de Dengue, Zika y Chikungunya, además de apuntar qué medios de comunicación los entrevistados más utilizan para encontrar tales conocimientos. Método: Se trata de un estudio transversal y descriptivo realizado a través de un cuestionario cualitativo cuantitativo a partir de un muestreo representativo. Se entrevistaron a 432 habitantes, de dieciséis barrios diferentes de Anápolis. Resultados: Se encontró una población en su mayoría joven y del sexo femenino, que supo presentar la forma de transmisión común a las tres enfermedades, pero el conocimiento del agente etiológico no fue satisfactorio, habiendo una confusión entre el mismo y el vector. En cuanto a los signos y síntomas, se observó mayor conocimiento de los participantes sobre el Dengue y aún un patrón de respuestas similar para las tres enfermedades lo que denota la falta de distinción entre ellas. Se notó que la población de Anápolis conoce y se ha adherido a las formas de control del mosquito Ae. Aegypti. Conclusión: De esta forma, se concluye que la población investigada posee conocimiento satisfactorio sobre los lugares de reproducción y las formas de prevención del mosquito, pero ese conocimiento parece no reflejar en sus actividades de prevención en domicilio, lo que contribuye al creciente número de personas casos de estas enfermedades. Por lo tanto, se hace evidente la necesidad de campañas educativas y mayor divulgación de información sobre Dengue, Zika y Chikungunya que aborden no sólo las medidas preventivas como los agentes etiológicos, el ciclo de la enfermedad, sus signos y síntomas y complicaciones.

Palabras clave: Aedes, Salud Pública, Comunicación en Salud.

\section{INTRODUÇÃO}

Os recentes surtos das doenças transmitidas pelo mosquito Aedes aegypti como a Dengue, a Zika e a Chikungunya se tornaram uma das principais preocupações não só da população brasileira como dos órgãos de governo que controlam a saúde, tendo repercussão em toda mídia nacional e internacional. Diante desses acontecimentos, a velocidade com que a informação é difundida passa a ter importância fundamental, através dos meios televisivos, escritos e até mesmo nas redes sociais, para que o governo possa se comunicar com a população no sentido de esclarecer os fatos, levar conhecimento e educar a sociedade. No entanto, quando se fala em saúde pública é importante compreender o processo pelo qual as informações atingem as comunidades, circulam e são interpretadas e internalizadas por seus integrantes, uma vez que, de maneira ágil ou não, seu contexto é essencial para construção de estratégias tanto de prevenção quanto no combate a doenças epidêmicas, como a Dengue, a Zika e a Chikungunya (VILLELA e ALMEIDA, 2012).

As ações de controle e prevenção da Dengue no Brasil, estiveram, no século passado, relacionadas às campanhas de erradicação do vetor Ae. aegypti. Após a década de 80 , sua dispersão geográfica foi observada em todo o país e desde então, os investimentos nessas campanhas de educação em saúde vêm crescendo. Mesmo assim nos últimos dois anos, houve o aparecimento e crescimento de duas novas endemias, a Chikungunya e a Zika. Em 2014, foram notificados 3.657 casos autóctones suspeitos de febre Chikungunya em oito cidades brasileiras (BRASIL, 2016). Já em 2015, foi confirmada transmissão autóctone de febre pelo vírus Zika, a partir do mês de abril, sendo que o ano se encerrou com a confirmação laboratorial de autoctonia da doença em 19 estados (BRASIL, 2016), com o agravante de estar relacionada à microcefalia em crianças.

REAS/EJCH | Vol.11 (4) | e274 | DOI: https://doi.org/10.25248/reas.e274.2019 Página 2 de 11 
Quanto à Dengue, foram notificados 3384 casos da doença em 2018 até a $48^{\mathrm{a}}$ semana epidemiológica que corresponde ao período de 31/12/2017 a 01/12/2018 segundo dados da Secretaria do Estado de Goiás (BRASIL, 2018).

Dessa forma, tem-se observado a necessidade de adequação dos planos de ação, visando o combate ao vetor de acordo com as necessidades de cada região brasileira. A população que já era vulnerável a quatro sorotipos da dengue, agora também está exposta a dois novos vírus, e essa é uma realidade compartilhada por inúmeras cidades brasileiras. As declarações de saúde internacionais têm enfatizado nas últimas décadas a importância da realização das ações educativas e de mobilização social na prevenção e controle de doenças, destacando a necessidade de se construir vigilância em saúde através da via sociocultural, onde toda a comunidade participa ativamente na mudança de comportamento e passa a adotar novas práticas de profilaxia. Assim, os conhecimentos adquiridos, bem como a percepção do risco ao qual a população está exposta, podem resultar em mudança de hábitos. Essa mudança de comportamento faz-se ainda mais importante quando envolve a eliminação dos criadouros para o Ae. aegypti no ambiente doméstico (MARTELLI, 2004; SOUSA, 2016).

Dentre as doenças transmitidas pelo Ae. aegypti está a Dengue que é manifestada, na maioria das vezes de forma benigna, com febre entre 39 e $40^{\circ}$, prostação, artralgias, cefaleias, dores musculares, manchas vermelhas na pele, que tem resolução entre três a sete dias, e astenia, que permanece por algumas semanas. No entanto, a doença pode evoluir com maior gravidade com sinais como petéquias, equimoses e hematêmese (FRANÇA et al., 2004; BRAGA e VALLE, 2007). Também pode ocorrer hemorragia gastrointestinal, assim como gengivorragia e epistaxe. Por fim, pode evoluir ao choque, a forma mais séria da doença, onde o paciente apresenta dor abdominal contínua, hepatomegalia dolorosa, vômitos, pele fria e pegajosa, taquicardia e oligúria. Em sua evolução, devido à acidose metabólica e coagulação intravascular disseminada, pode ocorrer o óbito em cerca de quatro a seis horas (CHAVES et al., 2016).

A Chikungunya que se caracteriza por artralgias simétricas, principalmente em tornozelos, dedos dos pés e cotovelos, de forte intensidade, que apresentam, na maioria das vezes, caráter debilitante, febre com mais de $38,5^{\circ} \mathrm{C}$, dores musculares e de cabeça. Os sintomas têm duração de cerca de 10 dias, porém, pode se estender por meses e até mesmo por anos, sendo caracterizada como um problema articular crônico. Alguns pacientes, principalmente crianças, idosos e adultos portadores de doenças crônicas como diabetes, asma, insuficiência cardíaca e alcoolismo, tem a propensão a desenvolver a forma grave da doença, devendo ser internados em unidades de terapia intensiva. Devido a suas características sintomáticas, que implicam muitas vezes na incapacidade do doente de realizar suas atividades diárias, é conferido a ela também, uma grande importância à saúde pública brasileira (CHAVES et al., 2016).

Já a Zika possui padrão de febre baixa, com menos de $38,5^{\circ} \mathrm{C}$, que dura de um a dois dias, ou ausência de febre, exantema pruriginoso por dois a sete dias, dor muscular de leve intensidade, dor e edema de articulações de intensidade inferior à encontrada na Chikungunya, e conjuntivite não purulenta. A probabilidade da associação do Zika vírus com a microcefalia foi o marco inicial de uma atenção diferenciada às gestantes portadoras do vírus. Tal associação foi confirmada pelo Center of Disease Control and Prevention, segundo o qual a presença do vírus é necessária para o agravo (FEBRASGO, 2016).

Todas as três doenças apresentam grande importância para a sociedade brasileira devido às suas sequelas, gastos públicos e reincidência. Entretanto, são enfermidades negligenciadas, com intervenções meramente tecnológicas ou biológicas, e assim apropriadas pelos meios de comunicação apenas em momentos de surtos epidêmicos, com informações a seu respeito propagadas na maioria das vezes de maneira incompleta (VALLE et al., 2016; CHAVES et al., 2016).

A fim de se adotar um planejamento voltado para o processo educativo, é preciso analisar o diagnóstico inicial da comunidade, investigar o conhecimento e a percepção da população a respeito do vetor e da doença, os hábitos e as características socioculturais relacionadas a estes. Assim, a partir desse conhecimento é possível focar em meios de comunicação específicos para atingir diferentes grupos na população e planejar estratégias educativas que atendam ao perfil das comunidades (SOUSA, 2016). 
Visando tal entendimento, este estudo tem como objetivo determinar o nível de conhecimento da população de Anápolis, Goiás, a respeito dos sintomas, sinais clínicos e formas de transmissão das doenças Dengue, Zika e Chikungunya, assim como sobre as formas de controle do mosquito vetor e comportamento da população na prevenção de tais doenças.

\section{MÉTODOS}

Trata-se de um estudo transversal e descritivo no qual foi aplicado um questionário semi-estruturado, de fácil entendimento à população em geral, na forma de entrevista, que permitiu uma avaliação objetiva sobre o conhecimento da população de Anápolis, Goiás, sobre o vetor, aspectos clínicos e medidas preventivas das doenças Dengue, Zika e Chikungunya. Para a caracterização dos sinais e sintomas corretos de Dengue, Zika e Chikungunya foi adotado que os sinais e sintomas: febre, dor por trás dos olhos, manchas no corpo, dor no músculo, dor de cabeça e dor na articulação eram sintomas comuns às três doenças. Enquanto que sangramento era um sinal próprio da Dengue e as opções microcefalia e conjuntivite específicos da Zika.

Os estudos transversais são estudos observacionais recomendados quando se deseja estimar a frequência com que um determinado evento de saúde se manifesta em uma população específica, além dos fatores associados com o mesmo e a coleta de dados pode envolver um recorte único no tempo (BASTOS e DUQUIA, 2007). Para a realização deste projeto, optou-se pela pesquisa quantitativa, a partir de uma amostragem representativa.

O estudo foi realizado em residências de 16 bairros de Anápolis-GO, previamente escolhidos por meio de sorteio. Os bairros sorteados foram Bandeiras, Cidade Jardim, Conjunto Habitacional Vila União, Itamaraty, Jardim Santa Cecília, Jundiaí, Maracanã, Parque Brasília, Parque Calixtópolis, Recanto do Sol, Santo Antônio, São João, Setor Industrial Aeroporto, Vila Celina, Vila Góis e Vila Jundiaí Industrial.

Para o cálculo do tamanho amostral levou-se em consideração que a população acima de 18 anos de Anápolis-GO é constituída por 226.875 pessoas. Neste o erro padrão de estimativa adotado foi de $5 \%$ e 0 nível de confiança de 95\% para o cálculo de uma população infinita como sugerido por Levin (2012).

A amostra calculada foi de 384 participantes.

A estratificação de Anápolis-GO foi feita com base em um mapa criado com dados do Google Earth no qual foi feita uma divisão da cidade em quatro regiões, de acordo com os pontos cardiais, tendo como o marco zero o cruzamento entre as avenidas Brasil Sul e Brasil Norte (sentido horizontal) e as Rodovias Federais BR330 e BR060 (sentido vertical) semelhante ao estudo realizado por Ferrari et al. (1998). A região 1 compreendeu a região sul-leste da cidade, a 2 sul-oeste, 3-norte-leste, 4-norte-oeste.

Cada quadrante foi dividido em bairros centrais e periféricos. Após esta divisão, a randomização dos bairros foi realizada via sorteio em envelopes lacrados. No total foram utilizados oito envelopes, dois para cada região, de modo a separar bairros centrais e periféricos. Foi solicitado ao orientador que retirasse dois bairros por cada envelope para compor os locais de coleta de dados, totalizando dezesseis bairros, sendo quatro bairros por quadrante.

Foram incluídos na pesquisa homens e mulheres, com 18 anos ou mais, de diferentes níveis socioeconômicos, que compreendam o teor das perguntas a serem feitas.

Foram excluídos da pesquisa os indivíduos que se recusem em responder alguma questão específica do questionário, os que não compreenderem o teor de alguma das perguntas, assim como os participantes que se sentirem constrangidos ou mesmo não quiserem continuar a entrevista, ainda que não exponham os motivos.

Os moradores foram recrutados em suas casas, através da exposição oral do projeto onde explicou-se todas as informações sobre a natureza, objetivos, procedimentos, riscos e benefícios dos participantes, assegurando o anonimato e sigilo das informações. A pesquisa realizada foi desenvolvida entre $8 \mathrm{~h}$ e $11 \mathrm{~h}$ da manhã e no período vespertino, entre $13 \mathrm{~h} 30 \mathrm{~min}$ e $17 \mathrm{~h} 30 \mathrm{~min}$, de segunda a sexta, conforme disponibilidade dos pesquisadores. 
Após a explicação sobre o estudo, aqueles indivíduos com interesse em participar da pesquisa assinaram o TCLE. Diante da recusa de participação, os pesquisadores se dirigirão às casas seguintes.

Os dados dos participantes foram coletados pelos pesquisadores através da aplicação de um formulário pré-elaborado e semiestruturado. Neste momento foram coletadas respostas que objetivam analisar 0 conhecimento dos indivíduos sobre sinais e sintomas das doenças Dengue, Zika e Chikungunya, o vetor e medidas de controle e prevenção destas.

O questionário aborda os seguintes eixos temáticos: dados pessoais e a percepção dos participantes sobre estas doenças, seu vetor e, por fim, as medidas para prevení-las.

O questionário utilizado foi adaptado de Sousa (2016).

Os resultados foram apresentados na forma de frequência simples e percentual. Os dados foram processados no programa MsExcel 2013 e analisados no pacote estatístico Statistical Package for Social Sciences (SPSS 21.0).

Esta pesquisa foi realizada de maneira objetiva, clara e respeitando sempre o indivíduo participante. Para isto está apoiada na Resolução 466/12 do Conselho Nacional de Saúde, onde são estabelecidos critérios para pesquisas que envolvem seres humanos. Como pesquisadores, deve-se visar a não-maleficência, beneficência, autonomia e justiça, a fim de resguardar e proteger os pesquisados.

O presente trabalho foi submetido e aprovado pelo Comitê de Ética em Pesquisa da Unievangélica, número do parecer 1.869.216.

\section{RESULTADOS}

Os dados foram coletados em dezesseis bairros de Anápolis, Goiás, representando 432 participantes. Quanto às características sociodemográficas dos entrevistados, a maioria era do sexo feminino, com a faixa etária entre 18 e 29 anos, e nível de escolaridade até o $2^{\circ}$ grau (Tabela 1).

Tabela 1. Características sócio-demográficas dos 432 participantes do inquérito domiciliar. Anápolis, Goiás, 2017.

\begin{tabular}{cc}
\hline \multicolumn{2}{c}{ Dados sociodemográficos } \\
\hline Sexo & $\mathbf{n}(\%)$ \\
Feminino & $267(61,8)$ \\
Masculino & $165(38,2)$ \\
\hline Grau de instrução & \\
1o grau $^{\circ}$ grau & $167(38,7)$ \\
Superior & $181(41,9)$ \\
Não sei & $83(19,2)$ \\
\hline
\end{tabular}

\begin{tabular}{cc}
\hline Faixa etária & \\
$18-29$ & $129(29,9)$ \\
$30-39$ & $107(24,8)$ \\
$40-49$ & $80(18,3)$ \\
$50-59$ & $61(14,1)$ \\
$>60$ & $55(12,7)$
\end{tabular}

Onde: $\mathrm{n}=$ frequência simples; \% = percentual.

Fonte: Dados da pesquisa, 2017 
A Tabela 2 refere-se ao conhecimento e percepção da população acerca de Dengue, Zika e Chikungunya, seus agentes etiológicos e formas de transmissão. Nesta, é possível perceber que a quase toda a população entrevistada (99,5\%) sabia o que era Dengue, enquanto com relação à Zika $(86,3 \%)$ e Chikungunya $(78,9 \%)$ esses números foram menores. Quanto ao agente etiológico das três doenças, a maioria relatou ser 0 mosquito, sendo que apenas $23,8 \%$ da população afirmou ser um vírus o causador da Dengue, $21,1 \%$ da Zika e $17,61 \%$ da Chinkungunya. Quanto à transmissão a opção "por mosquitos" foi a mais escolhida, sendo que na Zika 10,4\% escolheram a opção da transmissão por "contato sexual" e 22,2\% de "mãe para filho".

Tabela 2. Conhecimento e percepção de Dengue, Zika e Chikungunya, agente etiológico e formas de transmissão. Anápolis, Goiás, 2017.

\begin{tabular}{lccc}
\hline Perguntas e respostas & $\mathbf{n}(\%)$ & $\mathbf{n}(\%)$ & $\mathbf{n}(\%)$ \\
\hline Sabe o que é ... & Dengue & Zika & Chikungunya \\
Sim & $430(99,5)$ & $373(86,3)$ & $341(78,9)$ \\
Não & $2(0,5)$ & $59(13,7)$ & $91(21,1)$ \\
\hline Quem causa ... & Dengue & Zika & Chikungunya \\
Bactéria & $9(2,1)$ & $8(1,9)$ & $9(2,1)$ \\
Mosquito & $312(72,2)$ & $296(68,5)$ & $286(66,2)$ \\
Vírus & $103(23,8)$ & $91(21,1)$ & $76(17,6)$ \\
Não sei & $8(1,9)$ & $37(8,6)$ & $61(14,1)$ \\
\hline Transmissão ... & Dengue & Zika & Chikungunya \\
Contato sexual & $7(1,6)$ & $45(10,4)$ & $13(3)$ \\
Pelo ar & $8(1,9)$ & $13(3)$ & $6(1,4)$ \\
Por mosquitos & $424(98,1)$ & $388(89,8)$ & $353(81,7)$ \\
Pela água & $32(7,4)$ & $19(4,4)$ & $14(3,2)$ \\
De mãe para filho & $13(3)$ & $96(22,2)$ & $22(5,1)$ \\
Não sei & $1(0,2)$ & $36(8,3)$ & $73(16,9)$ \\
\hline
\end{tabular}

$\mathrm{n}=$ frequência simples; \% = percentual.

Fonte: Dados da pesquisa, 2017

Quanto aos sinais e sintomas (Tabela 3), para as três doenças, as opções mais apontadas foram febre e dor de cabeça. Enquanto os menos citados foram microcefalia $(11,8 \%)$ e conjuntivite $(4,4 \%)$ na Dengue, sangramento $(24,3 \%)$ e conjuntivite $(10 \%)$ na Zika e conjuntivite $(6,9 \%)$ e microcefalia $(19,7)$ na Chikungunya. Zika e Chikungunya ainda tiveram maior percentual de entrevistados que marcaram a opção "não sei" quanto aos aspectos clínicos da doença. Microcefalia foi uma opção apontada por 55,1\% dos participantes no questionário de Zika. 
Tabela 3. Conhecimento e percepção dos aspectos clínicos de Dengue, Zika e Chikungunya. Anápolis, Goiás, 2017.

\begin{tabular}{lccc}
\hline \multicolumn{1}{c}{ Sinais e sintomas } & Dengue & Zika & Chikungunya \\
\hline Febre & $\mathbf{n}(\%)$ & $\mathbf{n}(\%)$ & $\mathbf{n}(\%)$ \\
Dor por trás dos olhos & $425(98,4)$ & $344(79,6)$ & $305(70,6)$ \\
Manchas no corpo & $399(92,4)$ & $288(66,7)$ & $267(61,8)$ \\
Sangramento & $377(87,3)$ & $255(59)$ & $212(49,1)$ \\
Dor no músculo & $263(60,9)$ & $105(24,3)$ & $89(20,6)$ \\
Conjuntivite & $344(79,6)$ & $222(51,4)$ & $235(54,4)$ \\
Microcefalia & $19(4,4)$ & $43(10)$ & $30(6,9)$ \\
Dor de cabeça & $51(11,8)$ & $238(55,1)$ & $85(19,7)$ \\
Dor na articulação & $416(96,3)$ & $313(72,5)$ & $289(66,9)$ \\
Não sei & $365(84,5)$ & $281(65)$ & $287(66,4)$ \\
\hline
\end{tabular}

Onde: $\mathrm{n}$ = frequência simples; $\%$ = percentual.

Fonte: Dados da pesquisa, 2017.

A respeito do conhecimento da população sobre o vetor Aedes aegypti (Tabela 4), nota-se que os locais de reprodução do vetor: vasos ou plantas com água, caixa d'água destampada e depósitos com água foram apontados por mais de $90 \%$ dos participantes. Enquanto que "piscinas tratadas semanalmente" foi a opção indicada por apenas 5,3\% dos entrevistados. Quando perguntados se todo mosquito Aedes aegypti está infectado pelo agente etiológico dessas doenças, apenas $54,4 \%$ afirmaram que não e $56,7 \%$ dos entrevistados apontaram que a forma de contaminação do mosquito é picando uma pessoa contaminada.

Tabela 4. Conhecimento e percepção sobre o vetor Aedes aegypti. Anápolis, Goiás, 2017.

\begin{tabular}{lc}
\hline Perguntas e respostas & $\mathbf{n}(\%)$ \\
\hline Local de reprodução & $425(98,4)$ \\
Vasos ou plantas com água & $391(90,5)$ \\
Caixa d'água destampada & $424(98,1)$ \\
Depósitos com água & $23(5,3)$ \\
Piscinas tratadas semanalmente & $\mathbf{n}(\%)$ \\
Todo mosquito está com o agente etiológico & $180(41,7)$ \\
Sim & $235(54,4)$ \\
Não & $17(3,9)$ \\
Não sei & $\mathbf{n}(\%)$ \\
Como o mosquito se torna infectado & $105(24,3)$ \\
Quando ele nasce & $34(7,9)$ \\
No reservatório de água & $70(16,2)$ \\
De outro mosquito contaminado & $245(56,7)$ \\
Picando uma pessoa contaminada & $42(9,7)$ \\
Não sei &
\end{tabular}

Onde: $n$ = frequência simples; \% = percentual.

Fonte: Dados da pesquisa, 2017. 
A Tabela 5 representa o conhecimento dos participantes à respeito das medidas de prevenção de Dengue, Zika e Chikungunya e as medidas adotadas por eles em suas residências. Nota-se que medidas de combate à água parada como: "lavar periodicamente os reservatórios de água" e "destruir depósitos que juntem água" foram apontadas por mais de $90 \%$ dos entrevistados quando perguntados sobre as medidas de controle do vetor. Já em relação às medidas adotadas em suas residências, 96,5\% dos participantes disseram eliminar depósitos não utilizados de água, enquanto que apenas 2,1\% relatou não fazer nada para combater o Aedes aegypti.

Tabela 5. Conhecimento, percepção e adesão às medidas de prevenção das doenças. Anápolis, Goiás, 2017.

\begin{tabular}{lc}
\hline \multicolumn{1}{c}{ Medidas de controle do vetor } & $\mathbf{n}(\%)$ \\
\hline Lavar periodicamente os reservatórios de água & $423(97,9)$ \\
Usar telas & $385(89,1)$ \\
Usar inseticida & $321(74,3)$ \\
Destruir depósitos que juntem água & $393(91)$ \\
Não sei & $1(0,2)$ \\
Adesão às medidas & \\
Elimina depósitos não utilizados de água & $417(96,5)$ \\
Coloca areia nos pratinhos de planta & $305(70,6)$ \\
Usa inseticida & $271(62,7)$ \\
Não faço nada & $9(2,1)$ \\
Não sei & $0(0)$ \\
\hline
\end{tabular}

Onde: $\mathrm{n}$ = frequência simples; \% = percentual.

Fonte: Dados da pesquisa, 2017

Quanto aos meios de informação (Tabela 6) relatados pelos entrevistados houve destaque para a televisão representando $93,5 \%$ de respostas.

Tabela 6. Principais meios de informação sobre Dengue, Zika e Chikungunya. Anápolis, Goiás, 2017.

\begin{tabular}{lc}
\hline \multicolumn{1}{c}{ Meios de informação } & $\mathbf{n}(\%)$ \\
\hline Rádio & $196(45,4)$ \\
Televisão & $404(93,5)$ \\
Internet & $236(54,6)$ \\
Jornal e revista & $143(33,1)$ \\
Profissionais de saúde & $130(30,1)$ \\
Materiais educativos & $112(25,9)$ \\
Professores de escola/faculdade & $55(12,7)$ \\
\hline
\end{tabular}

Onde: $\mathrm{n}$ = frequência simples; \% = percentual.

Fonte: Dados da pesquisa, 2017. 


\section{DISCUSSÃO}

O presente estudo foi o primeiro realizado no município de Anápolis-GO nos moldes de inquérito domiciliar para avaliar o conhecimento e a percepção da população sobre a Dengue, Zika e Chikungunya, suas formas de transmissão, medidas de controle além das práticas de prevenção. A partir das 432 entrevistas realizadas, os resultados indicaram que a maioria dos participantes foram do sexo feminino, o que se explica pelo fato da cidade em questão apresentar uma população de maioria feminina (BRASIL, 2016), além disso, as mulheres estão em maior número presentes em seus domicílios durante o horário comercial, no qual foi aplicado a pesquisa, algo que corrobora com Gonçalves Neto et al. (2004), que observou $72,5 \%$ dos participantes do sexo feminino em sua pesquisa.

A faixa etária dos entrevistados indicou uma participação mais expressiva entre 18 e 39 anos (Tabela 1). Quanto à escolaridade, a maior porcentagem havia concluído o segundo grau, seguido de perto pelos que apresentavam o primeiro grau completo, revelando que a maioria trouxe conhecimentos sedimentados pelo ensino básico para responder ao formulário. Nascimento (2004) também concluiu em seus percentuais de estudo tal correlação positiva $(p<0,001)$ entre a percepção sobre a Dengue, Zika e Chikungunya e os participantes com segundo e terceiro grau de instrução.

Ao analisar a percepção da população sobre o nível de conhecimento dos entrevistados sobre essas três doenças, seus agentes etiológicos, formas de transmissão, aspectos clínicos e vetor é possível confrontar a realidade do que a população imagina que sabe e o que ela realmente conhece sobre estas doenças transmissíveis. Neste contexto, observou-se que os participantes ao serem perguntados se conheciam as doenças em questão, estes, com raríssimas exceções, disseram conhecê-las. Diante disso, a análise de alguns sintomas específicos merece atenção: dentro dos aspectos clínicos, os resultados indicaram que a maioria dos participantes possuem conhecimento satisfatório a respeito dos sintomas da Dengue clássica, porém isso não ocorre com a Zika e Chikungunya. Por exemplo, o sintoma de sangramento, um sinal de alerta para as formas hemorrágicas da Dengue, teve um resultado que mostrou um índice de acerto baixo, porém isso pode ser explicado pela exposição da população à infecção viral predominantemente da forma clássica da Dengue. Tal padrão de conhecimento, baseado em evidências, é também visto em países asiáticos. Neles os inquéritos que avaliaram o conhecimento da população determinaram a febre e a hemorragia como os principais sintomas reconhecidos pela sociedade, de modo que são encontrados nestes países altos coeficientes de incidência da forma hemorrágica, com grande impacto social e altas taxas de mortalidade infantil (NASCIMENTO, 2004).

Foi também observado que quando questionados sobre a Zika e a Chikungunya os participantes se mostraram incertos quanto aos sinais e sintomas. Dessa forma, as mesmas respostas foram as mais citadas nas três doenças pesquisadas, ou seja, febre, dor de cabeça e dor muscular, variando apenas na Zika, a qual microcefalia também foi um sinal bastante relatado (MARACAJÁ et al., 2016). Esta situação também pode ser explicada pela recente entrada dessas duas últimas doenças no cenário nacional, já que os primeiros casos somente foram relatados a partir de 2014 (BRASIL, 2016).

Quanto à pergunta "Quem causa" e "Quem transmite", os resultados exaltam a falta de conhecimento gerando uma confusão na população pesquisada, de modo que a maioria acredita que tanto quem causa quanto quem transmite as três patologias seja o mosquito. Algo também notório em relação a este tópico é o desconhecimento da população sobre as duas novas formas de transmissão da Zika, que são por contato sexual e a transmissão vertical, que apresentaram nesta pesquisa resultados muito baixos, demonstrando que há uma perigosa porta para a propagação desta doença e, provavelmente, uma comunicação ineficiente entre os meios de comunicação e a população (ARAÚJO, 2009).

A análise dos resultados encontrados em relação à transmissão mostrou níveis significativos de conhecimento sobre o vetor Ae. Aegypti na população estudada, assim como foi visto no estudo de Sousa (2016). Logo, o conhecimento referente ao local de reprodução do mosquito, medidas de controle e a adesão no combate ao vetor mostrou-se elevado, sendo que este constitui-se, não raro, o núcleo das mensagens veiculadas pelas campanhas educativas.

REAS/EJCH | Vol.11 (4) | e274 | DOI: https://doi.org/10.25248/reas.e274.2019 Página 9 de 11 
As medidas de prevenção divulgadas como essenciais para o controle da Dengue, como a eliminação de criadouros para o vetor no ambiente intradomiciliar e peridomiciliar, se mostram estar incorporadas como atividades de prevenção pela população, visto os altos percentuais de participantes que afirmaram adotar as medidas preventivas. Em confronto, Nascimento (2004) em estudo semelhante a este, no que tange às práticas preventivas de combate ao vetor nos domicílios, realizado no município de Goiânia-GO, a adesão às medidas preventivas não chegou a $50 \%$ da população estudada. Possivelmente essa diferença de adesão seja explicada pelo tempo cronológico entre as duas, o que demonstra que as campanhas realizadas neste período, como a vigente em Anápolis desde 2017 intitulada "Anápolis contra a Dengue", possam ter contribuído positivamente.

Vale ressaltar o tópico "usar inseticidas" como meio de prevenção das doenças, pois 271 participantes $(62,7 \%)$ responderam que utilizam, o que vai contra às estratégias do Ministério da Saúde, o qual considera inadequado o uso de aerossóis do grupo dos piretroides, por causar graves consequências no meio ambiente e interferir na eficácia dos programas de controle da Dengue, tornando o mosquito ainda mais resistente ao inseticida. Esse mesmo órgão preconiza o uso de inseticidas apenas com equipamentos específicos e por equipes das Secretarias Estaduais e Municipais de Saúde, treinadas para seu manuseio, o "fumacê". Enquanto isso, outros autores, como Paumgartten e Delgado (2016) afirmaram que os repelentes podem ser considerados adjuvantes em prevenir doenças transmitidas por artrópodes incluindo malária e arboviroses como febre amarela, Dengue, Chikungunya, Zika e encefalite do Nilo Ocidental.

Ressalte-se que apesar de a grande maioria dos entrevistados do município de Anápolis-GO relatarem que combatem o foco do mosquito em suas casas, na prática o aumento dessas três doenças não confirma tal postura. Essa percepção é corroborada pelo estudo de Cavalcante et al. (2007) no qual grande maioria dos entrevistados também demonstraram conhecimento correto da transmissão da doença e dos meios para reduzir a densidade de mosquitos, porém, a inspeção dos domicílios e peridomicílios feita por eles revelou uma quantidade enorme de potenciais criadouros para os vetores.

Quanto aos recursos de comunicação midiática, é certo que eles são responsáveis por transmitir as formas de percepção do mundo e de inter-relação com o universo atual (OLIVEIRA, 2000). No entanto, tornase claro muitas das vezes que a mídia privilegia as vozes detentoras de poder. Há muito conteúdo deturpado, afim de favorecer a interesses particulares, além de pouco espaço para a população se expressar (ARAÚJO, 2009). No presente estudo, quando interrogado à população de Anápolis-GO sobre em quais meios de comunicação se obteve informações sobre Dengue, Zika e Chikungunya, a maioria dos participantes citou a televisão, seguido de internet e rádio. As opções menos citadas foram professores da faculdade/escola, profissional de saúde (médico, enfermeiro, agente de saúde), material educativo (cartazes, panfletos, etc.), mostrando que mesmo com o advento da internet, a televisão ainda nos dias de hoje possui caráter decisivo nas informações recebidas pela população. Porém, o que vai contrário ao esperado de uma população de profissionais da área da saúde é a pouca participação dos mesmos no repasse de informações de saúde de qualidade, algo que já foi visto por Lima et al. (2014) ao identificar grandes problemas de comunicação no predominante modelo sacerdotal em que os profissionais da saúde, no caso, os dominantes da informação, repassa à população apenas aquilo que the tem interesse, gerando importantes problemas de saúde pública à sociedade.

Algumas estratégias podem ser utilizadas a fim de melhorar o conhecimento da população sobre tais doenças. Dentre elas, destaca-se a divulgação de informações a respeito destas enfermidades durante todo o ano, sem privilegiar épocas de epidemias. O repasse das informações deve ser feito de forma integral, priorizando os meios de divulgação mais utilizados por esta população, como televisão, internet, rádio, jornais e revistas. Ressalte-se que apesar de menos citados pela população na presente pesquisa, os profissionais de saúde podem aumentar sua participação neste repasse de informações, já que apresentam a facilidade de estarem inseridos no contexto regional, de forma específica. 


\section{CONCLUSÃO}

De acordo com este trabalho conclui-se que a população mostrou níveis satisfatórios de conhecimento sobre a Dengue e medidas de controle do vetor Aedes aegypti. Porém, o nível de conhecimento a respeito de Zika e Chikungunya se mostrou insatisfatório, principalmente no que se refere aos sintomas e formas de transmissão das doenças. Isso se verifica provavelmente pelo maior tempo de vigência da primeira doença citada no país, associado a incontáveis companhas do Ministério da Saúde e Secretarias municipais de Saúde. Porém, o desconhecimento sobre as doenças Zika e Chikungunya se torna preocupante, de modo que já se passaram quase três anos dos primeiros surtos no Brasil e o que se observa é apenas uma população confusa em relação a estas duas doenças ou em total falta de conhecimento das mesmas.

\section{REFERÊNCIAS}

1. ARAÚJO IS. Um olhar sobre as relações entre o SUS e a mídia. Seminário SUS 20 anos, Rio de Janeiro, Fiocruz, 2009; 62-69.

2. BASTOS JLD, DUQUIA RP. Um dos delineamentos mais empregados em epidemiologia: estudo transversal. Scientia Medica, 2007; 17(4): 229-232.

3. BRASIL. Ministério da Saúde. Boletim Epidemiológico. Secretaria de Vigilância em Saúde, Ministério da Saúde, 2016; 47(31). ISSN 2358-9450.

4. BRASIL. Ministério da Saúde. Boletim Epidemiológico. Secretaria de Vigilância em Saúde, Ministério da Saúde, 2018; 49(32). ISSN 2358-9450.

5. BRASIL. Ministério da Saúde. Boletim: Casos Notificados. Secretaria Estadual de Saúde: GO. 2018. Disponível em: https://extranet.saude.go.gov.br/public/dengue.html. Acesso em: 9 dez. 2018.

6. BRAGA IA, VALLE D. Aedes aegypti: histórico do controle no Brasil. Epidemiologia e serviços de saúde, 2007; 16(2): $113-118$.

7. CAVALCANTE KRJL, PORTO VT, TAUIL PL et al. Avaliação dos conhecimentos, atitudes, e práticas em relação à prevenção de dengue na população de São Sebastião-DF, Brasil, 2006. Comunicação em ciências da saúde, 2007; 18(2): 141-146.

8. CHAVES MRO, BERNARDO AS, BERNARDO CD et al. Dengue, Chikungunya e Zika: a nova realidade brasileira. Bio e Graphia, 2016.

9. FEBRASGO, Federação Brasileira das Sociedades de Ginecologia e Obstetrícia. Manual de Orientações e recomendações da FEBRASGO sobre a infecção pelo vírus Zika em gestantes e microcefalia. São Paulo, 2016.

10. FERRARI FP, FILHO NAR, RIBAS LFO et al. Prevalência de asma em escolares de Curitiba-projeto ISAAC (International Study of Asthma and Allergies in Childhood). Jornal de Pediatria, 1998; 74(4): 299-305.

11. FRANÇA E, ABREU D, SIQUEIRA M et al. Epidemias de dengue e divulgação de informações pela imprensa. Cadernos de Saúde Pública, 2004; 20(5):1334-1341.

12. GOOGLE. Google Earth. Version 7.1. 2015. Nota (Anápolis, Goiás).

13. GONÇALVES NETO VS, MONTEIRO SG, GONÇALVES AG et al. Conhecimentos e atitudes da população sobre dengue no Município de São Luís, Maranhão, Brasil, 2004. Cadernos de Saúde Pública; 2004; 22(10): 2191-2200.

14. LIMA CA, OLIVEIRA ANS, MACEDO BF et al. Relação profissional-usuário de saúde da família: perspectiva da bioética contratualista. Revista Bioética, 2014; 22(1): 152-160.

15. LEVIN, J. Estatística Aplicada a Ciências Humanas. São Paulo: Harbra Ltda. 2012.

16. MARACAJÁ MCS, SALES LR, DE SOUZA KLM et al. Dengue, chikungunya e zika vírus na escola: educação em saúde, 2016.

17. NETO FC; BARBOSA AAC, CESARINO MB et al. Controle do dengue em uma área urbana do Brasil: avaliação do impacto do Programa Saúde da Família com relação ao programa tradicional de controle. Cadernos de Saúde Pública, 2006; 22(5): $987-997$.

18. OLIVEIRA VC. A comunicação midiática e o sistema único de saúde. Interface-comunicação, saúde, educação, $2000 ; 71$ p.

19. PAUMGARTTEN FJR, DELGADO IF. Repelentes de mosquitos, eficácia para prevenção de doenças e segurança do uso na gravidez. Vigilância Sanitária em Debate: Sociedade, Ciência \& Tecnologia: 2016; 4(2): 97-104.

20. SOUSA LB. Estudo de infestação por Aedes aegypti na epidemiologia de dengue e percepção da população de Rio Claro, SP sobre aspectos da doença. 2016. 104f. Dissertação (mestrado) - Universidade Estadual Paulista, Instituto de Biociências de Rio Claro. Rio Claro, 2016.

21. VALLE D, PIMENTA DN, AGUIAR R et al. Zika, dengue e chikungunya: desafios e questões. Epidemiologia e Serviços de Saúde, Brasília; 2016; 25(2): 419-422.

22. VILLELA EFM, ALMEIDA MA. Mediações da informação em Saúde Pública: um estudo sobre a dengue. Revista Eletrônica de Comunicação, Informação \& Inovação em Saúde; 2012; 6(1). 